\title{
On the Performance of Transmuted Logistic Distribution: Statistical Properties and Application
}

\author{
Adeyinka Femi Samuel \\ Department of Mathematics, Obafemi Awolowo University, Ile-Ife, Nigeria. \\ fs.adeyinka247@gmail.com
}

\begin{abstract}
In this article we transmute the logistic distribution using quadratic rank transmutation map to develop a transmuted logistic distribution. The quadratic rank transmutation map enables the introduction of extra parameter into its parent model to enhance more flexibility in the analysis of data in various disciplines such as biological sciences, actuarial science, finance and insurance. The mathematical properties such as moment generating function, quantile, median and characteristic function of this distribution are discussed. The probability density functions of the minimum and maximum order statistics of the transmuted logistic distribution are established and the relationships between the probability density functions of the minimum and maximum order statistics of the parent model and the probability density function of the transmuted logistic distribution are considered. The parameter estimation is done by the method of maximum likelihood estimation. The flexibility of the model in statistical data analysis and its applicability is demonstrated by using it to fit relevant data. The study is concluded by demonstrating that the transmuted logistic distribution performs better than its parent model.
\end{abstract}

Keywords : logistic distribution; parameter estimation; order statistics; transmutation.

\section{Introduction}

Logistic distribution has been used over the years to model real life data in various fields of human endeavors such as medicine, Economics, Demography to mention a few. The probability density function (pdf) and cumulative distribution function (cdf) of logistic model are given by

$g(x)=\frac{e^{-\left(\frac{x-\mu}{\sigma}\right)}}{\sigma\left(1+e^{\left.-\left(\frac{x-\mu}{\sigma}\right)\right)^{2}}\right.} \quad,-\infty<x<\infty$

and

$$
G(x)=\left(1+e^{-\left(\frac{x-\mu}{\sigma}\right)}\right)^{-1},-\infty<x<\infty
$$

respectively where $\mu \leq x$ is the location parameter and $\sigma>0$ is the scale parameter.

The transmutation of some baseline distributions have been studied in the last decade to improve on the flexibility of the existing probability models. Shaw et al (2009) suggested a quadratic transmutation map and applied it to probability models such as exponential, uniform and normal distribution. Aryal et al (2009) worked on the transmuted extreme value distribution, Aryal et al (2011) on transmuted Weilbul distribution, Aryal et al (2013) on transmuted log-logistic, Merovci et al (2014) on transmuted Pareto distribution, Rahman et al (2018) generalized the work of Shaw et al (2009), Adeyinka et al (2019) on four parameters transmuted generalized distribution, Adeyinka et al (2019) on transmuted type I generalized half logistic distribution to mention a few. 
However, little or no consideration has been given to the transmutation of logistic distribution. This work intends to study the transmutation of logistic distribution, establish its properties and also consider the applicability of the model to real life data.

\section{Methodology}

\subsection{Transmuted Logistic Distribution}

If a random variable $\mathrm{X}$ has logistic distribution with probability density function given in

(1) and the cumulative distribution function given in (2). The corresponding transmuted logistic distribution, using the quadratic rank transmutation map,

$F(x)=(1+x) G(x)-\lambda G^{2}(x), \quad[\lambda] \leq 1$

is given by

$$
F(x)=\frac{1+(1+\lambda) e^{-x}}{\left(1+e^{-x}\right)^{2}},-\infty<x<\infty,
$$

and the corresponding pdf is obtained by differentiating (4) with respect to $x$ to have

$f(x)=\frac{e^{-x}\left\{(1+\lambda)\left(1+e^{-x}\right)-2 \lambda\right\}}{\left(1+e^{-x}\right)^{3}},-\infty<x<\infty$.

We assume $\mu=0$ and $\sigma=1$ with no loss of generality.

Figure 1 and 2 illustrate the graphs of pdf and cdf of transmuted logistic distribution for some selected values of parameter $\lambda$ respectively.

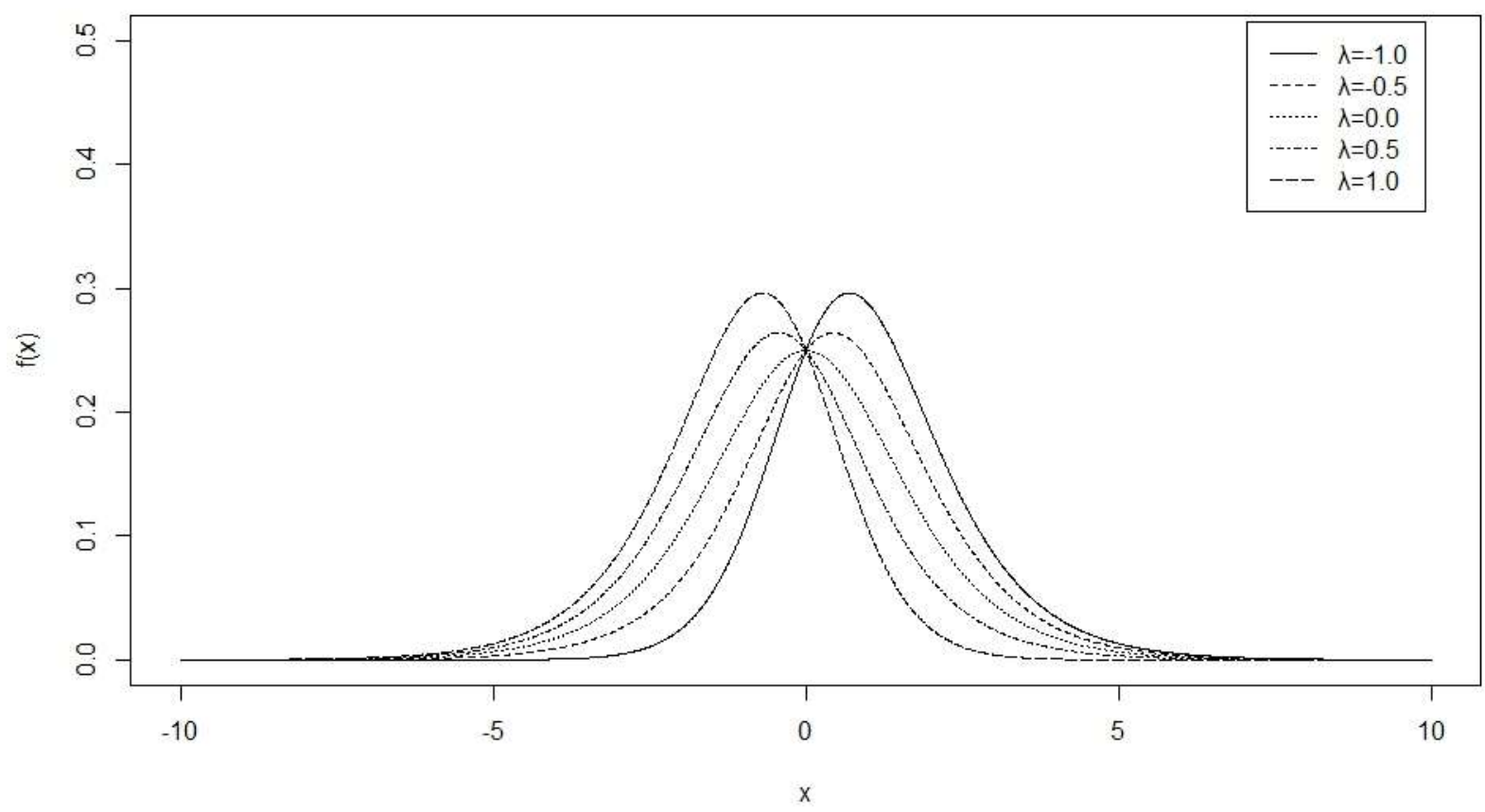

Figure 1: The probability distribution function of transmuted logistic distribution. 


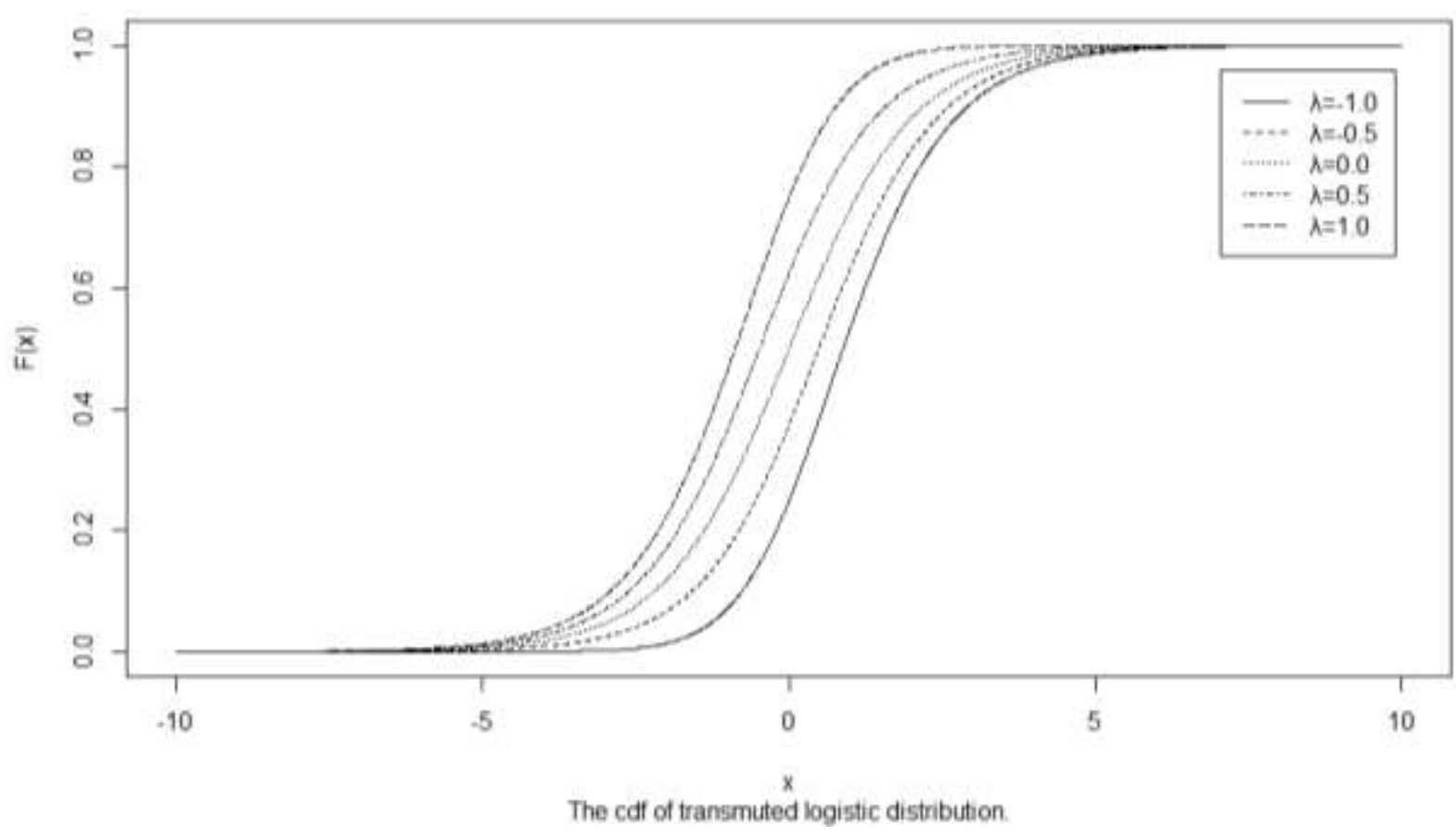

Figure 2: The cumulative distribution function of transmuted logistic distribution.

\subsection{Moment Generating Function of Transmuted Logistic Distribution}

The moment generating function of transmuted logistic random variable $X$, is given by

$M_{X}(\mathrm{t})=\mathrm{E}\left[e^{t X}\right]=\int_{-\infty}^{\infty} e^{t x} f(x) d x$

where $f(x)$ is the pdf of transmuted logistic distribution. This implies

$(1+\lambda) \int_{-\infty}^{\infty} \frac{e^{-x(1-t)}}{\left(1+e^{-x}\right)^{2}} d x-2 \lambda \int_{-\infty}^{\infty} \frac{e^{-x(1-t)}}{\left(1+e^{-x}\right)^{3}} d x$

Setting $y=e^{-x}, x=-\ln y$ and $d x=-\frac{1}{y} d y$. By substituting these into (7) it becomes

$$
(1+\lambda) \int_{0}^{\infty} \frac{y^{-t}}{(1+y)^{2}} d y-2 \lambda \int_{0}^{\infty} \frac{y^{-t}}{(1+y)^{3}} d y
$$

Setting $u=\frac{1}{1+y}, y=\frac{1-u}{u}$ and $d y=-\frac{1}{u^{2}} d y$. By substituting these into (8) it becomes

$(1+\lambda) \int_{0}^{1}(1-u)^{-t} u^{t} d u-2 \lambda \int_{0}^{1}(1-u)^{-t} u^{t+1} d u$

The integral in (10) is a beta function given by

$B(a, b)=\int_{0}^{1} x^{a-1}(1-x)^{b-1} d x$. 
$B(a, b)=\frac{\Gamma(a) \Gamma(b)}{\Gamma(a+b)}$ and $\Gamma(n)=(n-1) !$

Using these functional relationships (10) becomes

$(1+\lambda) \Gamma(1-t) \Gamma(1+t)-\lambda \Gamma(1-t) \Gamma(2+t)$

Similarly, the characteristic function transmuted logistic distribution is given by

$Q_{X}(i t)=(1+\lambda) \Gamma(1-i t) \Gamma(1+i t)-\lambda \Gamma(1-i t) \Gamma(2+i t)$

The mean, variance, skewness and kurtosis of the transmuted logistic distribution can be obtained from (11).

\subsection{Quantiles of Transmuted Logistic Distribution}

The $q^{t h}$-quantile of the transmuted logistic distribution is given by

$F_{X}\left(x_{q}\right)=q$

Where $F_{X}(x)$ is the cdf of transmuted logistic distribution. This implies

$\frac{1+(1+\lambda) e^{-x} q}{\left(1+e^{-x} q\right)^{2}}=q$

By solving for $x_{q}$ in (14) we have

$x_{q}=-\ln \left\{\frac{(1+\lambda-2 q)-\sqrt{(1+\lambda)^{2}-4 \lambda q}}{2 q}\right\}$.

The median of the transmuted logistic distribution is obtained by setting $q=0.5$ in (15) to have

$x_{0.5}=-\ln \left\{1+\lambda-\sqrt{1+\lambda^{2}}\right\}$

\section{Discussion}

\subsection{Number Generation from Transmuted Logistic Distribution}

Random numbers can be generated from transmuted logistic distribution by using the method of inversion as follows:

$F_{X}(x)=u$

Where $u \sim U(0,1)$ i.e $u$ is uniformly distributed on $(0,1)$ and $F_{X}(x)$ is the cdf of transmuted logistic distribution. Therefore, (17) implies

$\frac{1+(1+\lambda) e^{-x}}{\left(1+e^{-x}\right)^{2}}=u$ 
By solving (18) for $x$ it becomes

$x=-\ln \left\{\frac{(1+\lambda-2 u)-\sqrt{(1+\lambda)^{2}-4 \lambda u}}{2 u}\right\}$

Random numbers can be generated from (19) when the value of parameter $\lambda$ is known.

\subsection{Order Statistics from Transmuted Logistic Distribution}

We know that if $X_{(1)} \leq X_{(2)} \leq \cdots \leq X_{(n)}$ denotes the order statistics of a random sample $X_{1}, X_{2} \ldots X_{n}$ from a continuous population with cdf $F_{X}(x)$ and pdf $f_{X}(x)$, David 1970 gave the probability density function of $X_{(r)}$ as

$f_{X_{(r)}}(x)=\frac{1}{B(r, n-r+1)}[F(x)]^{r-1}[1-F(x)]^{n-r} f(x)$

where $r=1,2, \ldots n$.

We have from (1) and (2) the pdf of the $r^{\text {th }}$ order of the logistic random variable $X_{(r)}$ given by

$g_{X_{(r)}}(x)=\frac{e^{-x(n-r+1)}}{B(r, n-r+1)\left(1+e^{-x}\right)^{n+1}}$

Therefore the pdf of the $n t h$ order logistic statistic $X_{(n)}$ is given by

$g_{X_{(n)}}(x)=\frac{n e^{-x}}{\left(1+e^{-x}\right)^{n+1}}$

and the pdf of the $1^{\text {st }}$ order logistic distribution statistic $X_{(1)}$ is given by

$g_{X_{(1)}}(x)=\frac{n e^{-n x}}{\left(1+e^{-x}\right)^{n+1}}$.

Note that in a particular case of $n=2$, (22) yields

$g_{X_{(2)}}(x)=\frac{2 e^{-x}}{\left(1+e^{-x}\right)^{3}}$

and (23) yields

$g_{X_{(1)}}(x)=\frac{2 e^{-2 x}}{\left(1+e^{-x}\right)^{3}}$

It can be observed that $\max \left(X_{1}, X_{2}\right)$ and $\min \left(X_{1}, X_{2}\right)$ in (24) and (25) are special cases of (5) for $\lambda=-1$ and $\lambda=1$ respectively.

The distribution of the order statistics for the transmuted logistic distribution variable .

The pdf of the $r^{\text {th }}$ order statistics for the transmuted logistic distribution is given by 
$f_{X_{(r)}}(x)=\frac{e^{-x(n-r+1)}\left\{(1+\lambda)\left(1+e^{-x}\right)-2 \lambda\right\}\left\{1+(1+\lambda) e^{-x}\right\}^{r-1}\left\{1-\lambda+e^{-x}\right\}^{n-r}}{B(r, n-r+1)\left(1+e^{-x}\right)^{2 n+1}}$

Therefore the pdf of the largest order statistic $X_{(n)}$ is obtained when $r=n$ in (26) and it is given by

$f_{X_{(n)}}(x)=\frac{n e^{-x}\left\{(1+\lambda)\left(1+e^{-x}\right)-2 \lambda\right\}\left\{1+(1+\lambda) e^{-x}\right\}^{n-1}}{\left(1+e^{-x}\right)^{2 n+1}}$

and the pdf of the smallest order statistic $X_{(1)}$ is obtained when $r=1$ in (26) and it is given by

$f_{X_{(1)}}(x)=\frac{n e^{-n x}\left\{(1+\lambda)\left(1+e^{-x}\right)-2 \lambda\right\}\left\{1-\lambda+e^{-x}\right\}^{n-1}}{\left(1+e^{-x}\right)^{2 n+1}}$

\subsection{Estimation of Parameters from Transmuted Logistic Distribution}

Given a sample $X_{1}, X_{2} \ldots X_{n}$ of size $n$ from transmuted logistic distribution with the probability density function given by

$$
f(x ; \mu, \sigma, \lambda)=\frac{e^{-\left(\frac{x-\mu}{\sigma}\right)}\left\{(1+\lambda)\left(1+e^{-\left(\frac{x-\mu}{\sigma}\right)}\right)-2 \lambda\right\}}{\sigma\left(1+e^{-\left(\frac{x-\mu}{\sigma}\right)}\right)^{3}},-\infty<x<\infty
$$

where $\mu$ is the location parameter, $\sigma>0$ is the scale parameter and $|\lambda| \leq 1$ is the transmutation parameter.

The likelihood function of transmuted logistic distribution is given by

$$
L=e^{-\sum_{i=1}^{n}\left(\frac{x_{i}-\mu}{\sigma}\right)} \prod_{i=1}^{n}\left\{(1+\lambda)\left(1+e^{-\left(\frac{x_{i}-\mu}{\sigma}\right)}\right)-2 \lambda\right\} / \sigma^{n} \prod_{i=1}^{n}\left(1+e^{-\left(\frac{x_{i}-\mu}{\sigma}\right)}\right)^{3} .
$$

The log-likelihood function of (30) becomes

$$
\begin{aligned}
& \ln L=-n \ln \sigma-\sigma^{-1} \sum_{i=1}^{n}\left(x_{i}-\mu\right)-3 \sum_{i=1}^{n} \ln \left(1+e^{-\left(\frac{x_{i}-\mu}{\sigma}\right)}\right)+\sum_{i=1}^{n} \ln \{(1+\lambda)(1+ \\
& \left.\left.e^{-\left(\frac{x_{i}-\mu}{\sigma}\right)}\right)-2 \lambda\right\} .
\end{aligned}
$$

The maximum likelihood estimate of the parameters $\mu, \sigma$ and $\lambda$ that are contained within the transmuted logistic distribution function is obtained by differentiating (31) with respect to parameter $\mu, \sigma$ and $\lambda$ respectively and equating the result to zero as to have 


$$
\begin{aligned}
& \frac{\partial \ln L}{\partial \lambda}=\sum_{i=1}^{n} \frac{\left(1+e^{-\left(\frac{x_{i}-\mu}{\sigma}\right)}\right)-2}{\left.(1+\lambda)\left(1+e^{-\left(\frac{x_{i}-\mu}{\sigma}\right)}\right)-2 \lambda\right]}=0 \\
& \frac{\partial \ln L}{\partial \mu}=\sigma^{-1}\left\{n-3 \sum_{i=1}^{n} \frac{e^{-\left(\frac{x_{i}-\mu}{\sigma}\right)}}{\left(1+e^{-\left(\frac{x_{i}-\mu}{\sigma}\right)}\right)}+(1+\lambda) \sum_{i=1}^{n} \frac{e^{-\left(\frac{x_{i}-\mu}{\sigma}\right)}}{\left[(1+\lambda)\left(1+e^{-\left(\frac{x_{i}-\mu}{\sigma}\right)}\right)-2 \lambda\right]}\right\}=0 \\
& \frac{\partial \ln L}{\partial \sigma}=\sigma^{-2}\left\{-n \sigma+\sum_{i=1}^{n}\left(x_{i}-\mu\right)-3 \sum_{i=1}^{n} \frac{\left(x_{i}-\mu\right) e^{-\left(\frac{x_{i}-\mu}{\sigma}\right)}}{\left(1+e^{-\left(\frac{x_{i}-\mu}{\sigma}\right)}\right)}+(1+\right. \\
& \left.\lambda) \sum_{i=1}^{n} \frac{\left(x_{i}-\mu\right) e^{-\left(\frac{x_{i}-\mu}{\sigma}\right)}}{\left[(1+\lambda)\left(1+e^{-\left(\frac{x_{i}-\mu}{\sigma}\right)}\right)-2 \lambda\right]}\right\}=0
\end{aligned}
$$

The maximum likelihood estimator $\hat{\vartheta}=(\hat{\mu}, \widehat{\sigma}, \hat{\lambda})^{\prime}$ of parameters $\vartheta=(\mu, \sigma, \lambda)^{\prime}$ can be obtained by solving this non-linear system of equations. It is usually more convenient to use non-linear optimization algorithms such as quasi-Newton algorithm to numerically maximize the log-likelihood function in (31).

\subsection{Application of Transmuted Logistic Distribution}

The data used in this work is extracted from Gupta et al (2010). It is a strength data originally considered by Badar and Priest (1982). The data which represent the strength measured in GPA, for single carbon fibers and impregnated 1000-carbon fiber tows. Single fibers were tested under tension at gauge lengths of 1, 10, 20 and $50 \mathrm{~mm}$. Impregnated tows of 1000 fibers were tested at gauge lengths of 20,50,150 and $300 \mathrm{~mm}$. For illustrative purposes we are considering the single fibers data set of $10 \mathrm{~mm}$ in gauge lengths with sample size 63 . The data are presented below.

$1.901,2.132,2.203,2.228,2.257,2.350,2.361,2.396,2.397,2.445,2.454,2.474,2.518$, $2.522,2.525,2.532,2.575,2.614,2.616,2.618,2.624,2.659,2.675,2.738,2.740$, 2.856,2.917, 2.928, 2.937, 2.937, 2.977, 2.996, 3.030, 3.125, 3.139, 3.145, 3.220, 3.223, 3.235, 3.243,3.264, 3.272, 3.294, 3.332, 3.346, 3.377, 3.408, 3.435, 3.493, 3.501, 3.537, $3.554,3.562,3.628,3.852,3.871,3.886,3.971,4.024,4.027,4.225,4.395,5.020$.

A quasi Newton algorithm was implemented in $\mathrm{R}$ package and the performance of the models are shown in Table 1. Akaike Information criterion (AIC), Corrected Akaike Information criterion (AICC) and Bayesian Information criterion (BIC) were respectively used to compare the performance of transmuted logistic distribution (TLD) to its parent model (LD) in (1).

$$
\begin{gathered}
A I C=2 k-2 L L \\
A I C C=A I C+\frac{2 k(k+1)}{n-k-1}
\end{gathered}
$$

And 


$$
B I C=2 \log (n)-2 L L
$$

Where $\mathrm{k}$ is the number of parameters in the model, $\mathrm{n}$ is the sample size and LL is the maximized value of log likelihood function.

Table 1. Performance of the models.

\begin{tabular}{|l|l|l|l|l|l|}
\hline Model & Estimates & LL & AIC & AICC & BIC \\
\hline TLD & $\hat{\mu}=1.963$ & -112.789 & 231.578 & 231.985 & 229.177 \\
& $\hat{\sigma}=0.251$ & & & & \\
& $\hat{\lambda}=0.393$ & & & & \\
\hline LD & $\hat{\mu}=2.013$ & -121.452 & 246.904 & 247.104 & 246.503 \\
& $\hat{\sigma}=1.051$ & & & & \\
\hline & & & & & \\
\hline
\end{tabular}

It can be concluded from the results in table 1 that the transmuted logistic distribution (TLD) performs better than its parent model (LD).

\section{Conclusion}

In this article, we have introduced a new generalization of logistic distribution called transmuted logistic distribution. The distribution which is generalized by the use of quadratic rank transmutation map. Some statistical properties such as moment generating function, quantile, median and characteristic function of the transmuted logistic distribution are established and parameters estimation issues are addressed. The order statistics from the model are considered including the minimum and maximum order statistics and the relationships between the order statistics from the its base distribution and the probability density function of the transmuted logistic distribution for selected values of transmutation parameter $\lambda$ are demonstrated .The subject model is used to fit relevant data. We expect that this study will serve as a reference and help to advance future research in this area and other related disciplines.

\section{References}

Adeyinka F.S, and Olapade, A.K. (2019).On Transmuted Four Parameters Generalized LogLogistic Distribution. International Journal of Statistical Distributions and Applications. 5(2):32-37.

Adeyinka F.S, and Olapade, A.K. (2019). On the Flexibility of a Transmuted Type I Generalized Half-Logistic Distribution with Application. Engineering Mathematics. 3(1):13-18.

Aryal, G.R, and Tsokos, C.P. (2009). On the transmuted extreme value distribution with application. Nonlinear Analysis: Theory, Methods and Application.71 (12), el401-el407.

Aryal, G.R, and Tsokos, C.P. (2011). Transmuted Weilbull distribution: A generalization of Weilbull probability distribution. European Journal of Pure and Applied Mathematics. 4(2), 89-102.

Aryal, G.R. (2013). Transmuted log-logistic distribution. Journal of Statistics Applications and probability. 2(1),11-20. 
Badar, M.G. and Priest, A.M. (1982), "Statistical aspects of fiber and bundle strength in hybrid composites", Progress in Science and Engineering Composites, Hayashi, T., Kawata, K. and Umekawa, S. (eds.), ICCM-IV, Tokyo, 1129-1136.

David, H.A. (1970) Order Statistics. New York: Wiley Inter-science series.

Gupta, R.D., Kundu, D. (2010) .Generalized Logistic Distributions. Journal of Applied Statistical Science.18,51-66.

Merovci, F., Alizadeh, M., and Hamedani, G. (2016). Another Generalized Transmuted Family of Distributions: Properties and Applications. Austrian Journal of Statistics. 45, 71-93.

Merovci, F. (2014). Transmuted Generalized Rayleigh Distribution. Journal of Statistics Applications and Probability. 3(1), 9-20.

Merovci, F., Elbatal, I. (2014). Transmuted Lindley-geometric Distribution and its Applications. Journal of Statistics Applications and Probability. 3(1), 77-91.

Merovci, F., Puka, L. (2014). Transmuted Pareto Distribution. Probstat.7, 1-11.

Merovci, F. (2013). Transmuted Lindley Distribution. International Journal of open Problems in Computer Science and Mathematics. 6(2), 63-72.

O'Quigley, J., and Struthers, L. (1982). Survival model based upon the logistic andlog-logistic distribution. Computer programmes in Biomedicine. Vol 15. Pp 3-12.

Rahman M.M, Al-Zahrani B, Shahbaz M.Q (2018). A general transmuted family of distributions. Pak J Stat Oper Res 14:451-469.

Shaw, W.T, and Buckley, I.R. (2009). Alchemy of Probability Distributions: Beyond GramCharlier and Cornish -Fisher Expansions, and Skewed- kurtotic Normal Distribution from a Rank Transmutation Map. arxivpreprint arxiv: 0901.0434. 